\title{
Médiévales
}

Langues, Textes, Histoire

55 | automne 2008

Usages de la Bible

\section{Les marchands chassés du Temple, entre commentaires et usages sociaux}

The Cleansing of the Temple: Commentaries and Social Uses

\section{Emmanuel Bain}

\section{(2) OpenEdition}

\section{Journals}

Édition électronique

URL : http://journals.openedition.org/medievales/5449

DOI : 10.4000/medievales.5449

ISSN : 1777-5892

Éditeur

Presses universitaires de Vincennes

\section{Édition imprimée}

Date de publication : 20 décembre 2008

Pagination : 53-74

ISBN : 978-2-84292-221-4

ISSN : 0751-2708

\section{Référence électronique}

Emmanuel Bain, «Les marchands chassés du Temple, entre commentaires et usages sociaux », Médiévales [En ligne], 55 | automne 2008, mis en ligne le 20 mars 2011, consulté le 30 avril 2019. URL: http://journals.openedition.org/medievales/5449; DOI : 10.4000/medievales.5449

Ce document a été généré automatiquement le 30 avril 2019.

Tous droits réservés 


\title{
Les marchands chassés du Temple, entre commentaires et usages sociaux
}

The Cleansing of the Temple: Commentaries and Social Uses

\section{Emmanuel Bain}

\begin{abstract}
Jésus entra dans le Temple de Dieu. Il chassa tous ceux qui vendaient et qui achetaient dans le Temple; il renversa les tables des changeurs, et les sièges des vendeurs de colombes. Et il leur dit : Il est écrit : Ma maison sera appelée une maison de prière (domus orationis). Mais vous, vous en faites une caverne de voleurs ${ }^{1}$.
\end{abstract}

1 L'épisode célèbre des marchands chassés du Temple a fait l'objet, au cours du Moyen Âge, d'utilisations très variées ${ }^{2}$. Il a servi, entre autres, à condamner la simonie, à théoriser une saine colère, à valoriser les lieux de culte, à défendre les biens temporels de l'Église, à critiquer les marchands, à appeler à la Croisade, à justifier la violence contre les hérétiques, à blâmer les prêtres juifs, à dénoncer la cupidité, ou encore à appeler à une conversion intérieure. Il n'est pas question ici d'étudier en détail chacune de ces utilisations, mais de s'interroger sur la logique de leur formation. Nous nous demanderons si cette diversité est le résultat d'utilisations de convenances, arbitraires et subjectives, ou si elle s'ancre dans un travail d'interprétation plus profond et réfléchi ${ }^{3}$.

2 À cette fin, nous distinguerons deux types de textes : les commentaires, dont la fonction est d'expliquer le texte évangélique; et les utilisations, qui recourent au texte biblique dans le cadre de leur argumentation. Cette distinction ne signifie ni que les utilisations ne constituent pas des interprétations, ni que les commentaires ne sont pas motivés par des usages sociaux. Elle a une fonction essentiellement heuristique, dont cet article espère montrer l'intérêt en soulignant le rôle des commentaires dans les usages sociaux.

3 Après une rapide présentation des interprétations antiques de l'épisode des marchands chassés du Temple, nous soulignerons les grandes étapes qui renouvellent ses 
utilisations: les apports de Grégoire le Grand et de Bède, qui structurent les usages jusqu'à la réforme grégorienne; le changement de paradigme à la fin du $\mathrm{XI}^{\mathrm{e}}$ siècle ; puis l'introduction de la question des marchands au XII ${ }^{\mathrm{e}}$ siècle.

4 Les commentaires des Pères des premiers siècles forment le socle de l'interprétation de la péricope au cours du Moyen Âge. Trois aspects ressortent particulièrement: le cadre historique, les motivations du geste de Jésus, et les possibilités d'interprétations allégoriques.

5 Jérôme a livré l'explication historique de la présence des marchands dans le Temple : il s'agit, d'après lui, d'un système organisé par les prêtres juifs pour favoriser le culte et satisfaire leur avarice. Ceux-ci vendent aux pèlerins les animaux qui doivent être offerts en sacrifice, changent l'argent de ceux qui viennent de loin, et font crédit - moyennant un intérêt sous forme de menus cadeaux - à ceux qui en ont besoin ${ }^{4}$. Dans cette perspective, la virulence du geste de Jésus reste à expliquer : pourquoi met-il à bas une organisation vouée au culte? Plusieurs interprétations coexistent dans lesquelles se mêlent sens littéral et allégorique : la critique de l'avarice des prêtres, la condamnation du culte juif 5 , mais surtout - c'est le sens le plus courant de la péricope - la dénonciation du commerce des biens spirituels symbolisé par la vente des colombes ${ }^{6}$. Notons qu'aucun commentateur n'évoque la possibilité d'une hostilité de Jésus à l'égard du métier de marchand, ce qui reste une constante jusqu'au XII siècle.

Parallèlement, le Temple fait l'objet de diverses lectures allégoriques, notamment de la part d'Augustin : c'est la figure de l'Église, du corps ou de l'âme du fidèle, dont il faut chasser les vices ${ }^{7}$. Ainsi ce texte biblique ouvre-t-il à un discours général sur le bon comportement du chrétien.

7 Enfin, Augustin a harmonisé les Évangiles en expliquant que Jésus avait chassé par deux fois les marchands : une fois au début de sa vie publique, comme le rapporte Jean ; et une seconde fois à la fin, ce que relatent les Synoptiques ${ }^{8}$.

8 C'est sur un tel socle - une organisation liée au culte, un événement répété deux fois dans la vie du Christ, une condamnation de la vente des biens spirituels et la possibilité d'applications à la personne et à l'Église - que se bâtissent les interprétations médiévales.

9 Durant le haut Moyen Âge, deux auteurs font évoluer l'interprétation de manière fondamentale: Grégoire le Grand, puis Bède le Vénérable, dont les propos sont ensuite régulièrement repris.

10 Grégoire le Grand commente le passage des marchands chassés du Temple dans les homélies sur l'Évangile (homélies 17 et 39) prononcées au début de son pontificat, dans les années 590-592. Il reprend nombre d'aspects classiques du commentaire, mais apporte un élément nouveau essentiel, que résume un passage de la $17^{\mathrm{e}}$ homélie :

Mais notre Rédempteur renverse les sièges des vendeurs de colombes, parce qu'il réduit à rien le sacerdoce de tels marchands. Voilà pourquoi (hinc est quod) les canons sacrés condamnent l'hérésie simoniaque (simoniacam heresim) et prescrivent de priver du sacerdoce ceux qui accordent les ordres au prix d'une rémunération'.

11 Ce texte est important à double titre. C'est d'abord là que se forge l'expression d'« hérésie simoniaque». Simon, évoqué dans les Actes des Apôtres 8, 9-25, a tôt été considéré comme un hérétique, mais plus pour ses activités de magicien que pour sa tentative d'acheter l'Esprit Saint. La réflexion sur le lien entre Simon et la vente des biens spirituels se déployait séparément de celle relative à son caractère hérétique. Grégoire le Grand 
établit donc le lien entre l'hérésie et la simonie, avec l'expression d'«hérésie simoniaque ", qui devient courante, et efface, jusqu'au XI siècle, les légendes sur les pratiques magiques de $\operatorname{Simon}^{10}$.

Or Grégoire fonde cette expression sur la péricope des marchands du Temple : c'est parce que (hinc est quod) le Christ chasse les marchands que les canons condamnent l'« hérésie simoniaque ». Le raisonnement n'est pas développé, mais sa logique est aisée à reconstituer : le Christ chasse les vendeurs de colombes (c'est-à-dire les simoniaques), du Temple (c'est-à-dire de l'Église); donc les simoniaques doivent être chassés de l'Église, c'est-à-dire considérés comme hérétiques. Ce texte évangélique permet à Grégoire de définir une condamnation de la simonie plus rigoureuse que celle des Actes, et de la faire remonter au Christ et non aux seuls Apôtres ou aux canons. Par ailleurs, c'est de ce fondement biblique que nait la tension qu'analyse Charles de Miramon entre perspective contractuelle et hérétique, puisque la présence des marchands évoque le contrat tandis que le geste du Christ fonde l'accusation d'hérésie ${ }^{11}$.

13 Toutefois, la simonie est ici conçue par Grégoire dans le sens restreint de l'achat ou de la vente de l'ordination sacerdotale. En outre, il demande de priver de leur sacerdoce seulement ceux qui accordent les ordinations, pas ceux qui les achètent, alors que le Christ chasse acheteurs et vendeurs.

14 Après ce commentaire, à partir de 593, Grégoire diffuse largement l'expression d'« hérésie simoniaque », en la reprenant à vingt-sept reprises dans ses lettres, où il l'accompagne régulièrement de références aux marchands chassés du Temple ${ }^{12}$. L'influence de ces textes, régulièrement repris par les canonistes et les théologiens jusqu'au $\mathrm{XV}^{\mathrm{e}}$ siècle, est bien connue ${ }^{13}$. Toutefois, à l'époque carolingienne, les développements sur l'hérésie simoniaque en lien avec la péricope des marchands du Temple, sont bien moins fréquents que le discours sur le respect du lieu de culte qu'introduit Bède ${ }^{14}$.

15 Le deuxième changement important dans l'interprétation de ce passage est, en effet, apporté un siècle après Grégoire le Grand. Dans son commentaire sur Luc, Bède cite très longuement les homélies de Grégoire sans apporter d'éléments nouveaux ${ }^{15}$. Mais il en va différemment dans le commentaire sur Marc ${ }^{16}$ et dans une homélie sur Jean ${ }^{17}$. Dans ces textes, il met en place une interprétation du passage qui accorde une place importante à la question du lieu de culte ${ }^{18}$. Il note d'abord que Jésus, quand il entre à Jérusalem, se rend immédiatement au Temple, donnant ainsi un modèle de comportement :

Par le fait d'aller au Temple aussitôt entré dans la ville, il nous indique le modèle de piété (formam religionis) que nous devons suivre : quand il nous arrive d'entrer dans un domaine (villam), dans une place forte (oppidum), ou dans quelque autre lieu où se trouve une maison de prière consacrée à Dieu (domus orationis Deo consecrata), que nous fassions d'abord un détour vers ce lieu, et que seulement après nous être recommandés au Seigneur par l'exercice de la prière, nous nous livrions aux affaires temporelles pour lesquelles nous sommes venus ${ }^{19}$.

16 Par cette remarque, Bède souligne l'importance du lieu de culte dans la vie sociale. Il témoigne ainsi d'un processus de polarisation de l'espace par la maison de prière. Il définit par là, pour reprendre une expression de Michel Lauwers, une forme d' inecclesiamento: le lieu de culte est conçu comme un centre symbolique - pas nécessairement matériel : il faut se détourner pour aller à la maison de prière - de la vie sociale, même dans ses aspects les plus concrets (negocia temporalia) ${ }^{20}$. Cette importance suppose l'existence de lieux Deo consecrata $a^{21}$. À une époque où les rites de consécration 
sont assez sommaires, il ne faut probablement pas comprendre cette mention au sens technique, mais plutôt comme l'idée d'un lieu entièrement dédié à Dieu.

C'est pourquoi Bède insiste sur le respect du lieu de culte, dont doivent être exclues activités et pensées impures. Tel est le sens littéral et historique du texte. Au début de son homélie, après avoir expliqué, avec Augustin, que le commerce était d'autant plus licite qu'il consistait en offrandes pour Dieu, il en déduit que le problème est celui de sa présence in domo sua. Mais, contrairement à l'évêque d'Hippone qui, préoccupé par une mauvaise pratique des agapes, se contentait de fustiger la présence d'ivrognes dans les églises, Bède établit une série d'interdictions : ne doivent pas venir ceux qui sont pris dans des conflits (rixis dissidentes), ceux qui racontent des histoires (fabulis vacantes), ceux qui se tordent de rire (risu dissolutos), ou vaquent à d'autres crimes $^{22}$. Il ajoute ensuite une rapide réflexion sur la fonction du lieu, qui doit être un lieu de prière, et fustige ceux qui y ajoutent péchés sur péchés. Tout au long de ce passage, il suit le commentaire augustinien, dont il reprend de nombreuses expressions, mais en change radicalement le sens : là où le Père de l'Église traitait de péchés dans l'absolu, Bède dénonce leur présence au sein du lieu de culte.

Il revient à cette question à la fin de la péricope. Augustin expliquait que tout bon chrétien devait manifester son zèle pour la maison du Seigneur en corrigeant ses frères. Dans son texte, la maison du Seigneur ne désigne pas l'église, mais l'Église ${ }^{23}$. Bède, à nouveau, reprend de nombreuses formules augustiniennes, mais les applique cette fois clairement au bâtiment ecclésiastique : il établit une liste des vices capitaux et explique qu'il faut tout faire pour qu'ils n'y entrent pas. La pureté des personnes doit donc accompagner la pureté du lieu. Les raisons de ce respect du bâtiment ecclésiastique sont données : c'est le lieu où «le corps du Seigneur est consacré » et où l'« on ne peut douter de la présence perpétuelle des anges $»^{24}$. Cette présence angélique évoque la pierre de Jacob, elle-même symbole de l'autel. Ainsi ces deux images se rejoignent-elles pour faire de l'église un lieu à part, "consacré à Dieu», en raison de la présence du sacrifice eucharistique.

Bède semble donc le premier à interpréter la péricope des marchands chassés du Temple dans un sens qui fasse une place éminente à la défense du lieu de culte. Cette construction n'est pas isolée dans son œuvre: il a aussi été l'inventeur du genre des commentaires allégoriques sur le Temple ou le Tabernacle et a rédigé plusieurs sermons de dédicace ${ }^{25}$. Cette attention particulière aux lieux de culte s'inscrit dans le cadre d'un discours réformateur, qui appelle à la création de nouveaux diocèses et à la formation de meilleurs prédicateurs ${ }^{26}$. Scott DeGregorio a récemment souligné la présence des idéaux réformateurs dans l'exégèse de Bède, et insisté sur la volonté de combattre des institutions monastiques privées, qui seraient insuffisamment vouées au culte ${ }^{27}$. La réinterprétation de l'épisode des marchands du Temple confirme cette approche, qui montre la part des usages sociaux dans le commentaire.

20 Cette péricope, associée aux trois aspects du commentaire de Bède - polarisation de l'espace par l'église, respect du lieu de culte, et présence du sacrifice eucharistique constitue un des principaux fondements de la réflexion médiévale sur le lieu de culte ${ }^{28}$. Elle est reprise dans les commentaires bibliques: Alcuin cite intégralement les passages de l'homélie de Bède ${ }^{29}$; Raban Maur ${ }^{30}$ ou Christian de Stavelot ${ }^{31}$ citent le commentaire sur $^{2}$ Marc. Paschase Radbert évoque le respect dû au bâtiment fait de pierres, même s'il insiste plus sur l'interprétation spirituelle concernant l'Église faite de pierres vivantes ${ }^{32}$. L'interprétation proposée par Bède influence aussi la rédaction des textes normatifs 
soucieux d'établir le principe de la vénération des lieux de culte. L'Admonitio generalis (789) utilise ainsi la péricope pour interdire négoces et vains discours, dans un chapitre entièrement consacré au respect dû au lieu de culte ${ }^{33}$. Ces prescriptions influencent divers canons d'évêques ${ }^{34}$. L'interprétation se retrouve également dans des textes liés à la formation des laïcs. Dans le De institutione laicali, Jonas d'Orléans cite littéralement les passages de l'homélie de Bède, même s'il y ajoute des passages augustiniens qui instaurent un respect du lieu de culte lié à l'écoute de la parole de Dieu transmise par le prédicateur, plutôt qu'à la présence du sacrifice eucharistique ${ }^{35}$. Une génération plus tard, Hincmar de Reims, dans le De cavendis vitiis et virtitubis exercendis, établit, grâce à la citation de Bède, le lien entre respect du mystère eucharistique et respect du lieu où il

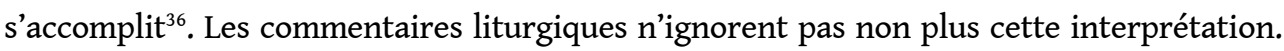
Walafrid Strabon consacre deux chapitres au respect de l'église, qu'il fonde entièrement sur l'épisode des marchands chassés du Temple ${ }^{37}$.

21 Néanmoins, apparait à l'époque carolingienne une évolution notable, qui réside dans l'élargissement de la question du respect du lieu à celle de la protection des res ecclesiae. Dans une épître adressée par Raban Maur, en qualité d'archevêque de Mayence, à Louis le Germanique en 847, il annonce qu'il va traiter de la révérence due aux églises (reverentia aecclesiarum), renvoie alors au geste du Christ qui a chassé du Temple les activités illicites, mais s'appuie sur ce texte pour appeler à la protection des biens temporels des églises et demander l'immunité, au nom du zèle que le roi doit manifester pour défendre les "églises du Christ $»^{38}$. Par ce passage du respect du lieu du sacrifice eucharistique, au respect des possessions des églises, Raban accompagne l'évolution de la signification du mot ecclesia ${ }^{39}$ et se place dans la continuité de divers textes carolingiens dans lesquels la péricope évangélique avait été utilisée dans ce contexte : le De dispensatione d'Agobard de Lyon, qui fustigeait la vente des églises et de ses possessions - crime plus grave que celui de la vente dans les églises rejetée par Jésus ${ }^{40}$; le traité, probablement rédigé par Jonas d'Orléans en 836 , qui indiquait que «notre rédempteur n'avait pas supporté la violation et le déshonneur (violationem et dehonorationem) infligés à son temple ${ }^{41} »$.

Après l'époque carolingienne, le geste de Jésus demeure une référence pour penser la sacralité du lieu de culte. Elle est fondamentale dans les actes du synode d'Arras ${ }^{42}$, présente dans le Contra Petrobrusianos de Pierre le Vénérable ${ }^{43}$ et d'autres traités antihérétiques ${ }^{44}$. Elle se retrouve encore au XIII ${ }^{\mathrm{e}}$ siècle dans de nombreux actes de synodes anglais ${ }^{45}$ et dans des sommes de confesseurs ${ }^{46}$.

23 Le lien de la péricope avec l'eucharistie et la pureté du lieu, pourrait expliquer sa représentation sur l'antependium doré de l'autel de Milan ( $1^{\text {re }}$ moitié du $\mathrm{IX}^{\mathrm{e}}$ siècle), et sur des lieux marquant un seuil, comme les portes de bronze de Saint-Zénon à Vérone ( $\mathrm{XII}^{\mathrm{e}}$ siècle) ou la façade de l'abbaye de Saint-Gilles-du-Gard (xII ${ }^{\mathrm{e}}$ siècle). Dans ce dernier cas, le choix de cette scène semblerait renforcer l'hypothèse d'un programme iconographique anti-hérétique. Mais sa présence peut aussi bien s'expliquer dans le cadre des autres hypothèses de lecture de la façade : discours contre les juifs, appel à la croisade, discours sur les richesses ${ }^{47}$. C'est du moins ce que montrent les usages de la péricope à partir du XI siècle.

24 Un livre d'Évangiles, réalisé en Italie du Nord à la fin du xI ${ }^{\mathrm{e}}$ siècle, et ayant peut-être appartenu à la comtesse Mathilde de Canossa, contient, en frontispice de l'Évangile de Jean, une image du Christ chassant avec son fouet une multitude de marchands, changeurs, prêteurs et d'animaux (voir image ci-dessous). La présence de cette scène en un lieu où elle apparaît comme une synthèse de l'Évangile johannique est à la fois originale et 
surprenante. Compte tenu du contexte réformateur dans lequel elle a été produite, il est tout à fait possible d'y voir, à la suite de Robert Rough, un symbole de la réforme - le Christ étant en l'occurrence le type de Grégoire VII chassant de l'Église simoniaques et laïcs ${ }^{48}$. Un tel usage de la péricope se retrouve effectivement dans les textes, qui permettent toutefois de distinguer deux périodes : dans un premier temps, les usages de la péricope biblique restent très dépendants de Grégoire le Grand, tandis qu'à partir du pontificat d'Urbain II, ils se renouvellent nettement et se diversifient.

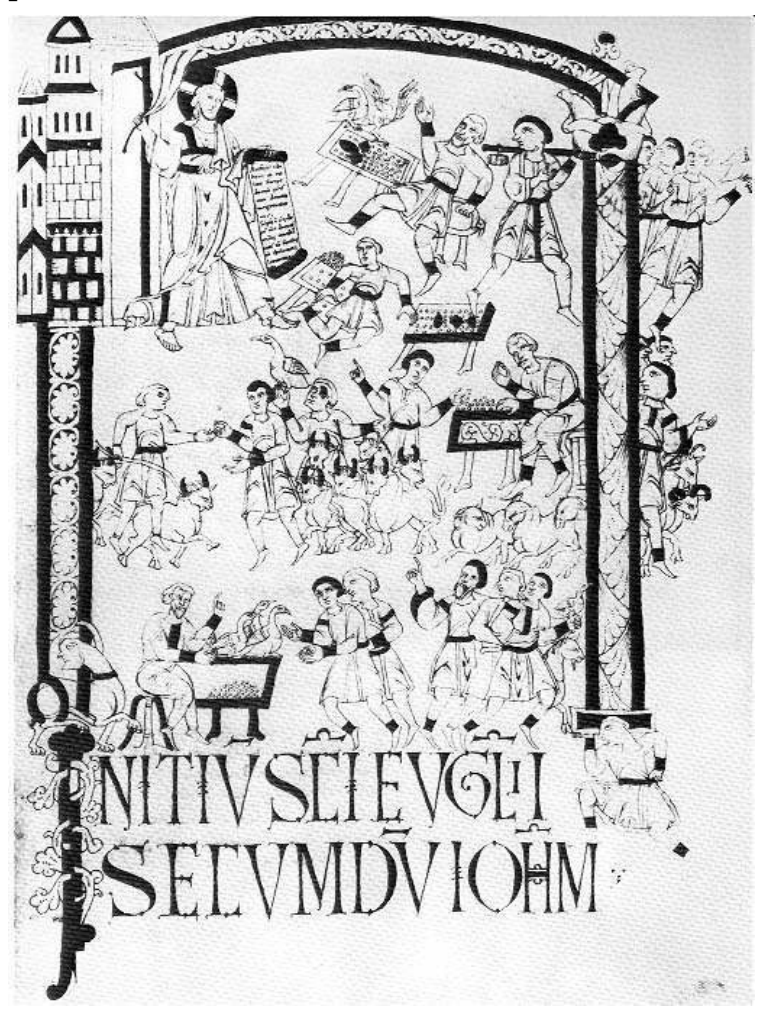

Jésus chassant les marchands du Temple (New York, Morgan Pierpont Library 492, 7.84r., cliché de l'auteur)

Il est bien connu que la simonie fait l'objet d'intenses réflexions au cours du $\mathrm{xI}^{\mathrm{e}}$ siècle et que l'expression d'«hérésie simoniaque » se diffuse très largement. Néanmoins, les usages de la péricope des marchands du Temple ne se renouvellent que très lentement. L'œuvre d'Humbert de Silva Candida en est une illustration. Il recourt à plusieurs reprises à l'image des marchands chassés du Temple, mais l'emploie toujours en l'associant à des citations de Grégoire, Augustin ou Remi d'Auxerre ${ }^{49}$, si bien qu'il dénonce à travers ce texte la simonie comme achat de l'ordination, alors qu'il construit par ailleurs une conception beaucoup plus large de la simonie qui intègre les biens temporels de l'Église ${ }^{50}$. De même, alors qu'Humbert s'attache à fonder théologiquement l'accusation d'hérésie contre les simoniaques ${ }^{51}$, il n'applique jamais ces réflexions à la péricope des marchands du Temple. À la génération suivante, Bernold de Constance, dans son commentaire des décrets de Grégoire VII, utilise lui aussi la péricope uniquement dans le cadre de l'interprétation de Grégoire le Grand ${ }^{52}$.

Le cas de Pierre Damien témoigne d'une légère évolution. Il utilise à trois reprises ce texte biblique comme symbole de la réforme : dans la lettre 13, adressée à Grégoire VI en 1045, il est cité parmi d'autres images bibliques pour évoquer le renouveau de l'Église; dans la lettre 20, c'est l'empereur qui est rapproché du Christ pour avoir révoqué un évêque 
simoniaque ; plus nettement, la fameuse lettre 40 (Liber gratissimus) s'ouvre par un poème sur les simoniaques qui renvoie à cette image ${ }^{53}$. Toutefois Pierre Damien utilise aussi le même texte biblique pour justifier la validité des sacrements conférés par les simoniaques, ce qui introduit une perspective bien moins réformatrice ${ }^{54}$. Il semble donc nécessaire de nuancer l'idée selon laquelle Pierre Damien aurait fait de ce texte biblique sa métaphore favorite de la réforme ${ }^{55}$.

Enfin, si l'on admet avec John Gilchrist que la plupart des clercs - en dehors d'Humbert de Silva Candida - ne considéraient pas véritablement la simonie comme une hérésie, mais plutôt comme un péché grave ${ }^{56}$, alors il faut admettre que les réformateurs employaient très fréquemment l'expression " hérésie simoniaque » à cause de l'autorité de Grégoire le Grand qui s'y trouvait attachée. Que cette autorité ait constitué un argument pour exiger la révocation des prélats simoniaques, une limite pour étendre la notion de simonie, ou un obstacle face au développement d'une conception contractuelle de la simonie, dans tous les cas, les usages du texte biblique en sont très dépendants.

Cette situation change totalement à l'époque d'Urbain II. Charles de Miramon a récemment montré le tournant que constitue ce pontificat pour la politique antisimoniaque $^{57}$. Le même constat peut être dressé pour les utilisations de l'épisode des marchands chassés du Temple, qui s'intègrent dans de nouveaux cadres : la défense des biens temporels de l'Église, l'action réformatrice, la définition d'un pouvoir coercitif de l'Église, et l'appel à la croisade. Dans une lettre de la fin de son pontificat adressée au prévôt Lucius, Urbain II défend à son tour l'idée que la vente des biens ecclésiastiques est une forme de simonie. Il s'appuie pour cela sur deux textes devenus classiques (l'Epistula Widonis, attribuée à Pascal I ${ }^{\text {er }}$, et le quatrième canon du concile de Chalcédoine), mais il va plus loin en recourant au passage des marchands du Temple. Probablement conscient d'introduire ainsi un nouvel usage de la péricope - jusque-là réservée à la condamnation des ordinations simoniaques ${ }^{58}$-, il éprouve le besoin d'affirmer que «tous les commentateurs » ont compris que les colombes désignaient les biens temporels (plus exactement l'intendance), et cite Augustin, que du reste il déforme ${ }^{59}$. Cette lettre connaît une ample diffusion, et à partir d'elle, il devient banal de justifier l'intégration des biens ecclésiastiques parmi les biens spirituels grâce à l'épisode des marchands du Temple.

Le deuxième changement de cette époque se situe dans l'amplification de l'appel à la réforme. Alors que Grégoire le Grand mettait en garde les chrétiens dans la perspective eschatologique du retour du Christ, Pierre Damien a commencé à utiliser ce passage pour un appel à la réforme hic et nunc. Peu avant Urbain II, Manegold de Lautenbach a exprimé cette idée bien plus clairement et fermement, en expliquant qu'il ne faut pas seulement chasser ceux qui vendent les saints offices, mais montrer qu'ils sont déjà bannis ${ }^{60}$. $\mathrm{Ce}$ changement de perspective temporelle a son pendant à la fin $d u \mathrm{XI}^{\mathrm{e}}$ siècle dans la conception des fonctions ecclésiastiques. Dans un sermon attribué à Yves de Chartres, le Christ chassant les marchands du Temple remplace comme modèle du clerc portier le Christ gardien de l'arche de Noé ou de la porte des enfers ${ }^{61}$. Ici aussi il y a passage d'une action dans l'au-delà à une action ici-bas. Or c'est précisément ce changement que donne à voir l'image des Évangiles de Mathilde. Sur l'antependium en or de Milan, le Christ est représenté à l'intérieur du bâtiment dans lequel se tiennent des disciples et dont il chasse les marchands ; sur la reliure du Codex aureus de Saint-Emmeran de Ratisbonne (IX siècle) ${ }^{62}$, plus nettement encore sur un ivoire conservé à Würzburg (vers 900) ${ }^{63}$, le Christ est à l'extérieur et se dirige avec un fouet vers le Temple dont il va chasser les marchands; dans une représentation des Évangiles d'Otton $\mathrm{III}^{64}$, le Christ est plus proche de la porte, 
mais a encore le bâtiment devant lui ; dans les Évangiles de Mathilde, le Christ est sans ambiguïté représenté en position de portier: de l'église qui est dans son dos n'est représenté que le porche, qu'il a pour fonction de garder. Cette caractéristique, tout autant que la possession du manuscrit par Mathilde de Canossa, manifeste la portée réformatrice que l'image attribue à la péricope.

La réforme peut être, comme le geste du Christ, violente. Dans son Décret, Yves de Chartres utilise un texte d'Augustin (extrait du Contra litteras Petiliani) qui qualifie le Christ chassant les marchands de persecutor, et qui distingue la persécution des justes, déplorable, de celle des injustes, qui est légitime ${ }^{65}$. En soi cette interprétation n'est donc pas nouvelle, mais d'une part Yves fait un montage de textes d'Augustin qui renforce la légitimation de la violence contre les hérétiques ${ }^{66}$, et d'autre part il introduit ce passage dans un ensemble discursif voué à l'affirmation du pouvoir coercitif de l'Église qui lui donne une nouvelle portée ${ }^{67}$. Ce texte étant repris dans le Décret de Gratien, l'idée d'associer la persecutio aux marchands du Temple se diffuse - même si d'autres passages bibliques sont plus souvent utilisés dans cette perspective.

Les nouvelles approches de la péricope aboutissent à son utilisation dans la prédication de croisade. D'après la version de Guillaume de Tyr, dans la seconde moitié du XII ${ }^{\mathrm{e}}$ siècle, Urbain II s'y serait référé dans l'appel de Clermont, dénonçant l'occupation du Temple par de petits démons ${ }^{68}$. La figuration possible des marchands chassés du Temple sur le linteau du Saint-Sépulcre va dans le même sens ${ }^{69}$. Dans son éloge des Templiers, Bernard en fait une longue utilisation où le Christ apparaît comme le chef des chevaliers, qui sert de modèle à cette nouvelle armée qui juge «beaucoup plus indigne et infiniment plus intolérable de laisser les infidèles souiller le saint lieu que de voir des marchands l'infester ${ }^{70} »$. L'ensemble du paragraphe repose sur l'assimilation littérale du Temple d'où Jésus avait chassé les marchands au lieu où sont installés les Templiers, ce qui rejoint plusieurs discours tendant à assimiler le Dôme du Rocher au Temple, dans le cadre de l'instauration d'un culte du Templum Domini ${ }^{71}$. D'ailleurs les pèlerins pouvaient y admirer la pierre sur laquelle le Christ avait posé le pied quand il chassait les marchands ${ }^{72}$.

L'époque de la réforme grégorienne révèle donc à la fois le poids de l'autorité du commentaire de Grégoire le Grand et ses limites. Jusqu'à la fin du siècle, elle détermine très largement les usages de la péricope des marchands chassés du Temple. Puis, sous le pontificat d'Urbain II, au moment où le modèle de la simonie fixé par Grégoire le Grand éclate - par un élargissement qui inclut dans la simonie l'achat ou la vente des biens ecclésiastiques, et par une disjonction entre l'hérésie et la simonie ${ }^{73}$ - les usages de la péricope se renouvellent. Les clercs invoquent alors (avec plus ou moins de rigueur) d'autres autorités; mais ce renouvellement semble surtout porté par l'évolution des conceptions de l'Église. L'usage de la péricope pour appeler aux croisades porte à son aboutissement une évolution d'une étonnante cohérence, caractérisée d'une part par un processus d'inscription spatiale de l'Église, qui assimile l'Église aux églises, puis aux biens d'églises, et enfin - moins nettement - à la Terre sainte, et d'autre part par un processus complémentaire de sécularisation du modèle christique, qui passe d'une menace dans l'au-delà, à un appel à l'action contraignante ici-bas. Ce schéma permet d'expliquer l'évolution exactement parallèle des usages de la figure de Simon, décrite par Herbert E.J. Cowdrey, qui s'étonne de la lenteur du renouveau de l'image de Simon comme magicien - effacée par l'interprétation de Grégoire le Grand - et constate lui aussi un tournant à la fin du siècle ${ }^{74}$. 

plus diversifiés que jamais. Elle intervient dans les discours sur la colère ${ }^{75}$, sur le judaïsme ${ }^{76}$, sur la simonie ${ }^{77}$, sur l'avarice des $\operatorname{clercs}^{78}$, sur le bon usage des biens ${ }^{79}$. Les lectures historiques s'approfondissent ${ }^{80}$, tandis qu'apparaissent des interprétations théologiques nouvelles ordonnées autour du Christ ${ }^{81}$. De ce vaste ensemble, nous ne retiendrons que deux aspects : le message transmis par la prédication, qui nous ramènera à la question du lieu; et ce qui forme le tournant majeur de l'interprétation de ce passage évangélique : l'introduction de la problématique des marchands. Jésus et le métier de marchand. Ce lien apparaît subitement, avec une virulence à la hauteur de celle du Christ, dans un texte - une palea - ajouté au Décret de Gratien dans la seconde moitié du XII ${ }^{\mathrm{e}}$ siècle, au plus tard à l'époque du décrétiste Huguccio, qui le cite. Le texte lui-même est extrait de l'Opus imperfectum in Mattheum, texte arien du ve siècle, mais qui était alors attribué à Jean Chrysostome ${ }^{82}$. Il s'ouvre par une phrase appelée à devenir célèbre : «En chassant les vendeurs et les acheteurs du Temple, le Seigneur a montré qu'un marchand ne peut jamais, ou à peine, plaire à Dieu (homo mercator vix aut nunquam potest Deo placere). C'est pourquoi nul chrétien ne doit être marchand [... $]^{83}$ ». La suite du texte condamne la vanité de la richesse du marchand qui ne sert pas dans l'audelà, puis distingue l'artisan (dont l'activité est honnête) du marchand (qui ne transforme pas les biens). Il présente ensuite un cas pire que celui du marchand: l'usurier, qui doit être bien dissocié du bailleur. Enfin, un dernier paragraphe évoque la suite du texte biblique ("Et il a renversé les tables des changeurs »), qu'il interprète allégoriquement: les seules pièces qui doivent être présentes dans le temple de Dieu sont les hommes qui, tels de bonnes pièces, portent sur eux l'image de Dieu.

Giacomo Todeschini a proposé une stimulante lecture de ce texte, refusant d'y voir une hostilité rédhibitoire de l'Église face au développement du commerce, mais plutôt une réflexion sur le rôle des clercs dans la société. Cette interprétation repose toutefois sur le contexte dans lequel apparaît la palea et sur son étude intégrale ${ }^{84}$. Or le texte circule aussi en dehors du Décret, et se trouve rarement cité dans son intégralité. Il exprime donc bien, dans les faits sinon dans l'intention de ceux qui l'ont adjoint au Décret, une pensée hostile au métier de marchand. Dès lors, compte tenu de l'ample diffusion du Décret d'une part, de l'Opus imperfectum d'autre part, le lien entre discours sur les marchands et utilisation $\mathrm{du}$ passage évangélique devient fréquent. Il se retrouve dans les commentaires canoniques, les commentaires bibliques, les commentaires des Sentences, les sommes de confesseurs, ou encore les sommes théologiques.

Comment expliquer l'apparition soudaine de cette nouvelle problématique? Probablement, comme l'a montré G. Todeschini, prend-elle sa source dans la réflexion grégorienne sur la distinction entre clercs et laïcs, puisque c'est dans ce contexte qu'apparaît pour la première fois nettement cette nouvelle interprétation. Il est aussi raisonnable de penser que le développement du commerce et des échanges est à l'origine d'un nouveau regard ecclésiastique sur les textes bibliques traitant des marchands. Plus encore, le développement d'une société marchande urbaine, construisant une culture propre, et des pouvoirs concurrents dans la cité, ont pu susciter, de la part des clercs, une nouvelle lecture d'un épisode biblique fameux, mais jusque-là essentiellement compris en terme de simonie. Plusieurs éléments confirment cette hypothèse. D'abord, l'homélie de l' Opus imperfectum sur ce passage biblique était connue à l'époque carolingienne, puisqu'il subsiste quelques manuscrits qui la contiennent ${ }^{85}$. De plus, une phrase de Léon le Grand 
( Il est difficile que dans le commerce entre un acheteur et un vendeur n'intervienne pas le péché ») était très connue, et aurait pu être appliquée aux marchands du Temple. Enfin, il existait bien quelques textes qui ne traitaient pas directement de l'épisode des marchands du Temple, mais où ils ont pu être associés à une critique des marchands ${ }^{86}$. Il est donc manifeste que les clercs disposaient d'un matériau suffisant pour construire une critique du métier de marchand, qu'ils auraient pu appliquer à cette péricope. Il semble donc que si les marchands ne sont pas pris en considération, c'est que cela ne répondait pas à une préoccupation des clercs plus embarrassés par des potentes envahisseurs de biens ecclésiastiques, que par des mercatores peu menaçants. Or cette situation évolue au cours du XII siècle.

Il est toutefois possible de faire une autre lecture de l'introduction de la problématique des marchands, qui tiendrait plutôt à l'histoire des textes et à la conception de l'autorité. L'hypothèse serait alors que l'introduction de la problématique des marchands ne résulterait pas d'une préoccupation sociale des clercs, mais de la redécouverte d'un texte doté du prestige et de l'autorité d'un Père grec. Notons d'abord que la découverte du passage de l'Opus imperfectum sur les marchands chassés du Temple n'est pas isolée : c'est l'ensemble de l'œuvre qui fait l'objet d'une « redécouverte » à partir du XII siècle. Ainsi dans les commentaires bibliques sur les Évangiles, Chrysostome est régulièrement cité à partir des années 1230 sur divers types de passages, qui n'ont pas nécessairement de rapport avec les activités économiques. L'étude de la tradition manuscrite, menée par Joop Van Banning, a bien montré que c'est à partir de la seconde moitié du XII siècle que se diffuse ce commentaire. Enfin, le fait même que le passage sur les marchands du Temple ait été ajouté dans le Décret de Gratien dans la seconde moitié du XII ${ }^{\mathrm{e}}$ siècle confirmerait que c'est seulement à partir de cette époque que cette œuvre est mieux connue.

De plus, la radicalité de l'Opus répond mal aux besoins et aux intentions des clercs. Ils ne souhaitent pas condamner le commerce, mais l'encadrer pour réinsérer la richesse produite dans un circuit économique contrôlé par les clercs qui, eux, se tiennent à l'écart du commerce. Leur intention n'est nullement d'exclure les marchands, mais au contraire de les faire pleinement participer à une société chrétienne ${ }^{87}$. En expliquant qu'un chrétien ne peut être marchand, le texte attribué à Chrysostome s'oppose à cette vision de la société, et embarrasse les clercs plus qu'il ne vient conforter leurs stratégies de contrôle social. C'est ce dont témoigne la grande majorité des commentaires qui mettent en place diverses stratégies discursives pour écarter la mise en cause de la pratique commerciale.

Huguccio ne commente pas la palea elle-même, mais s'y réfère régulièrement à propos de l'usure (c. 14, q. 3). Les usages qu'il en fait sont très éclairants : il y renvoie pour montrer que la negociatio est interdite aux clercs, que la transformation des biens - l'artisanat n'est pas une negociatio et qu'elle est donc permise aux clercs et aux laïcs, que la location n'est pas une usure. Huguccio ne retient donc de la palea que ce qu'elle ne condamne pas et l'interdiction du commerce faite aux clercs. Parallèlement il s'efforce de montrer que le commerce est permis aux laïcs, même sans transformer le bien vendu, en raison du service rendu ${ }^{88}$. Enfin, quand il traite des dangers du commerce, il ne renvoie pas à la palea, mais à la phrase de Léon le Grand reprise dans le De penitencia (d.5) ${ }^{89}$. Or cette phrase est non seulement bien moins sévère que celle de l'Opus imperfectum, mais elle s'intègre en outre dans un contexte où il est question de péchés véniels. C'est d'ailleurs ainsi qu'Huguccio le comprend dans le commentaire du De penitentia, où il explique que la 
marchandise est un métier honnête, même si des péchés peuvent être commis ${ }^{90}$. Le célèbre canoniste s'efforce avant tout de supprimer l'aspect anti-mercantile de l'opus imperfectum, dont il n'applique les proscriptions qu'aux clercs.

Dans sa Summa confessorum des années 1210, Thomas de Chobham semble éprouver des difficultés comparables. Dans le chapitre de clericis negotiantibus, il traite de la question du commerce en ayant manifestement sous les yeux le Décret de Gratien, et tout son travail consiste justement à détourner la condamnation radicale, en utilisant notamment l'argument de l'utilité sociale ${ }^{91}$. Globalement, ce que l'on pourrait appeler la rédemption du marchand est une constante des textes des canonistes, de Rufin au XIII ${ }^{\mathrm{e}}$ siècle $^{92}$.

41 Les commentaires bibliques, dans leur diversité, semblent témoigner d'une certaine hésitation. La Postille dominicaine, dans les années 1230, reprend la critique des marchands sur Matthieu, la présente de façon plus ambiguë sur Marc, la passe sous silence sur Luc, et l'intègre dans un discours sur le bon usage des richesses dans le commentaire de Jean ${ }^{93}$. De même, Nicolas de Goran ne fait pas allusion aux marchands sur Matthieu et Jean, mais cite Chrysostome sur Luc et Marc ${ }^{94}$. Alexandre de Halès semble reprendre à son compte l'opus imperfectum ${ }^{95}$, alors que son disciple Jean de La Rochelle l'interprète clairement dans un sens qui interdit le commerce seulement aux clercs ${ }^{96}$.

Le rapprochement entre la péricope évangélique et la problématique des marchands semble donc davantage résulter de la découverte d'un nouveau texte doté d'une grande autorité que des préoccupations sociales des clercs. Néanmoins, une fois le rapprochement établi, tout le travail des théologiens et canonistes consiste à intégrer cette nouvelle autorité dans le cadre des usages sociaux qu'ils peuvent en faire. C'est pourquoi leur effort porte d'une part sur l'affirmation de la licéité du commerce contre Chrysostome - et d'autre part sur l'interdiction du commerce pour les clercs. Comme l'a montré G. Todeschini, la représentation des marchands chassés du Temple par Giotto dans la chapelle des Scrovegni à Padoue, qui « oublie » les changeurs et l'argent, illustre le résultat de ce travail de commentaire des autorités ${ }^{97}$.

L'examen de quelques sermons du XIII ${ }^{\mathrm{e}}$ siècle rappelle par ailleurs que les usages de cette péricope ne se limitent pas à la question des marchands, et que les interprétations allégoriques conservent un rôle social de premier plan $^{98}$. Des réflexions sur la fonction du lieu de culte, et le respect qui lui est dû en conséquence, apparaissent régulièrement. Johannes de Castello explique qu'il faut se rendre à l'église parce que Dieu s'y trouve, parce que c'est un lieu plein de dévotions, et pour y recevoir les sacrements ${ }^{99}$. Augustinus Triomphus de Ancona estime que ce doit être le lieu de la prédication, des sacrements et de la prière ${ }^{100}$. Guillaume Peyraut précise que l'église doit être un lieu aimé, vénéré et dans lequel le fidèle place sa confiance ${ }^{101}$. Bonaventure évoque lui aussi trois fonctions : la purgation, l'illumination, la réfection sacramentelle ${ }^{102}$. Toutefois, le même Bonaventure, dans un autre sermon sur le même thème, n'évoque aucunement l'église, mais seulement la prière, expliquant que c'est elle qui bâtit la demeure de Dieu ${ }^{103}$. Le dominicain Aldobrandinus de Toscanella a laissé deux sermons sur ce thème. Dans l'un, destiné à une dédicace d'église, il commence par expliquer le sens de la consécration, qui est de faire de l'église un lieu apte à la prière, et passe immédiatement à de longs développements sur l'importance de la prière ${ }^{104}$. Dans l'autre, probablement adressé à des frères, il n'évoque pas le bâtiment ecclésiastique, mais seulement l'assemblée des religieux comme domus orationis $^{105}$. Nombreux sont les sermons qui n'évoquent le bâtiment que dans son sens allégorique. C'est le cas de Guibert de Tournai, qui construit l'intégralité de son sermon sur une identification du Temple à l'âme du fidèle ${ }^{106}$. De même, Eustache d'Arras évoque 
six allégorisations du Temple (le corps, la conscience, l'Église militante, la prière, le cloître, la patrie céleste), mais pas l'église ${ }^{107}$. La place accordée au bâtiment est rarement importante. Ces choix révèlent la multiplicité des usages possibles de l'image du Temple, mais ne signifient pas l'existence d'une opposition entre une église faite de pierres et de bois, et une église faite de pierres vivantes. Comme l'indique Guillaume Peyraut, «l'église extérieure est la figure des deux autres [la patrie céleste, l'âme intérieure], dévoilant ce qui est caché dans l'église supérieure, et les vertus qui doivent se trouver dans l'église inférieure ${ }^{108} »$. La sainteté des fidèles est un élément essentiel de la sainteté du lieu de culte: c'est ce qu'affirme Eustache d'Arras dans son sermon de dédicace ${ }^{109}$, rejoignant ainsi le message du synode d'Arras.

La péricope des marchands chassés du Temple constitue un cas exemplaire pour illustrer les usages sociaux de la Bible au Moyen Âge. Leur diversité ne signifie pas une instrumentalisation arbitraire de la Bible, mais s'ancre dans le système médiéval de production du sens du texte biblique, fondé sur la pluralité des significations et le recours aux autorités. La théorie des quatre sens explique que peuvent se superposer des utilisations pour appeler à la croisade (qui relève d'une interprétation historique), à la purification intérieure (sens tropologique), à la lutte contre la simonie (sens allégorique), ou à préparer le retour du Christ (sens anagogique).

Par ailleurs, ce passage a joué un rôle clé dans le développement de trois problématiques majeures du Moyen Âge : l'inscription de l'Église dans des lieux matériels (les églises et leurs possessions) ; la réflexion sur la simonie ; le rôle des marchands dans la société. Or ces trois cas montrent l'importance des commentaires qui, à chaque fois, précèdent les usages. L'homélie de Grégoire le Grand est à l'origine de la définition de l'hérésie simoniaque; celle de Bède précède et accompagne le processus de polarisation de la société autour des églises; c'est le commentaire, redécouvert au XII siècle et attribué à Chrysostome, qui ouvre la voie au discours sur les marchands. Mais les autorités peuvent aussi figer, pour un temps, les usages d'un texte : c'est ce qui semble se produire au début de la réforme grégorienne. Enfin, certains moments semblent plus propices que d'autres aux redéploiements des usages sociaux d'un texte : l'époque de Grégoire et de Bède, celle d'Urbain II.

Le travail quotidien du commentaire, au cœur de la construction cléricale du savoir et de l'autorité, est ainsi l'école où s'enseigne l'art de forger une pensée neuve ou socialement pertinente, à partir de textes anciens, si bien que dans la société, comme dans les textes, la Bible semble d'abord dotée d'une « fonction animatrice »"10, celle d'un lieu de réflexion, de construction d'un sens toujours évolutif, point de départ d'une reprise infinie dans laquelle utilisateurs et commentateurs font naître le texte à son temps.

\section{NOTES}

1.Mt 21, 12-13. Les quatre Évangélistes (Mc 11, 15-17 ; Lc 19, 45-46, Jn 2, 13-17) rapportent cet épisode, avec quelques différences introduites notamment par Jean, qui le situe au 
début de la vie publique de Jésus - et non après l'entrée triomphale à Jérusalem - et qui cite le Ps 69, $9:$ : «L zèle de ta maison me dévore ».

2.Je remercie vivement Michel Lauwers et Isabelle Rosé pour leur lecture attentive et leurs diverses suggestions.

3.Cet article voudrait se situer au croisement de deux approches de la Bible au Moyen Âge : l'approche sociale, illustrée notamment par Ph. Buc (L'Ambiguïté du Livre. Prince, pouvoir, et peuple dans les commentaires de la Bible au Moyen Âge, Paris, 1994) ou G. LOBRICHON (La Bible au Moyen Âge, Paris, 2003) ; et l'approche herméneutique conduite par G. DAHAN ( L'Exégèse chrétienne de la Bible en Occident médiéval XII ${ }^{e}$-XIV ${ }^{e}$ siècle, Paris, 1999).

4.JÉRôME, Commentaire sur s. Matthieu, II, É. BONNARD éd., Paris, 1979 (SC 259), p. 111-117. 5.AMBRoISE DE MILAN, Traité sur l'Évangile de s. Luc II, G. TISSOT éd., Paris, 1976 (SC 52b), p. 148.

6.AMBROISE, éd. cit., p. 147 ; AUGUSTIN, Homélies sur l'Évangile de saint Jean I-XVI, M.F. BERROUARD éd., Paris, 1993 (BA 71), p. 560-564 ; HILAIRE DE POITIERS, Sur Mathieu, II, J. DOIGNON éd., Paris, 1979 (SC 258), p. 126-9.

7.0p. cit. p. 570-571 ; Enarrationes in Psalmos CI-CL, E. DEKKERS, I. FRAIPONT éd., Turnhout, 1990 (CCSL 40), p. 1898-1900 (Psaume 130).

8.De consensu Evangelistarum, PL 34, 1141.

9.GRÉGOIRE LE GRAND, Homélies sur l'Évangile Livre I, 17, 3, R. ÉTAIX, C. MOREL, B. JUDIC éd., Paris, 2005 (SC 485), p. 385.

10.E. AMANN, «Simon le magicien », Dictionnaire de théologie catholique 14/2, 2130-2140 ; A. BRIDE, « Simonie », ibid., 14/2, 2141-2160 ; J. LECLERCQ, « Simoniaca heresis », Studi Gregoriani, 1, 1947, p. 523-530 ; H.E.J. COWDREY, « Simon Magus in South Italy », AngloNorman Studies, 15, 1992, p. 77-90.

11.ch. DE MIRAmON, «Spiritualia et Temporalia-Naissance d'un couple », Zeitschrift Der Savigny-Stiftung für Rechtsgeschichte, Kanonistische Abteilung, 92, 2006, p. 224-287 (p. 256-259).

12.Dans cinq lettres, situées entre 595 et 599 , se retrouve un raisonnement identique, qui associe l'hérésie simoniaque à la péricope des marchands du Temple $(\mathrm{V}, 58 ; \mathrm{V}, 62 ; \mathrm{V}, 63$; VI, 7 ; IX, 219) ; les mêmes idées sont aussi présentes en XI, 28 (601) ; mais dans les autres cas, l'expression d'hérésie simoniaque est employée incidemment, sans raisonnement spécifique pour l'accompagner. Cf. Registrum epistularum, D. NORBERG éd., Turnhout, 1982 (CCSL 140, 140 A).

13.J. LECLERCQ, « Simoniaca heresis », cit.; P. DE VOOGHT, « La "simoniaca heresis" selon les auteurs scolastiques ", Ephemerides Theologicae Lovanienses, 30, 1954, p. 64-80 ; J. GILCHRIST, "'Simoniaca Haeresis' and the Problem of Orders from Leo IX to Gratian », dans S. KUTTNER, J.J. RYAN éd., Proceedings of the Second International Congress of Medieval Canon Law, Vatican, 1965 (Monumenta Iuris Canonici, C, 1), p. 209-235.

14.Le rapprochement se trouve dans les commentaires bibliques sur Mathieu de RABAN MAUR (EXpositio in Matthaeum, B. LÖFSTEDT éd., Turnhout, 2000 (CCCM 175), p. 547-552), de CHRISTIAN DE STAVELOT (PL 106, 1432-1433), de PASCHASE RADBERT (Expositio in Matheo libri XII , B. PAULus éd., Turnhout, 1984 [CCCM 56B], p. 1023-1027 [p. 1026-1027]). Mais il semble rare dans les textes normatifs : en dehors du concile de Meaux de 845 (MGH, Capit. Reg. Franc. Occ. 2, p. 408), l'expression d'hérésie simoniaque circule dans un autre contexte scripturaire.

15.In Lucae Evangelium expositio, D. HURST éd., Turnhout, 1960 (CCSL 120), p. 347-350.

16.In Marci Evangelium expositio, D. HURST éd., Turnhout, 1960 (CCSL 120), p. 575-580. 
17.Homeliarum Evangelii libri II, D. HURST éd., Turnhout, 1955 (CCSL 122), p. 184-192.

18.Les quelques réflexions sur le lieu de culte présentes dans cet article se situent dans la continuité des recherches de D. IOGNA-PRAT (La Maison-Dieu. Une histoire monumentale de l'Église au Moyen Âge, Paris, 2006), et M. LAUWERS (Naissance du cimetière. Lieux sacrés et terre des morts dans l'Occident médiéval, Paris, 2005).

19.In Marcum, p. 575.

20.Sur l'inecclesiamento : M. LAUWERS, Naissance du cimetière..., op. cit., p. 273.

21.Cette construction d'un espace polarisé par des lieux mis hors du monde rappelle les analyses d'A. GUERREAU, «Quelques caractères spécifiques de l'espace féodal européen », dans N. BULST, R. DESCIMON, A. GUERREAU éd., L'État ou le roi. Les fondations de la modernité monarchique en France (XIV ${ }^{e}$-XVII siècles), Paris, 1996, p. 85-101 ; ID., " Structure et évolution des représentations de l'espace dans le haut Moyen Âge occidental », dans Uomo e spazio nell'alto medioevo. Settimane di studio del Centro italiano di studi sull'alto Medioevo, 50/1, Spolète, 2003, p. 91-115.

22.Homélie II, 1, p. 185.

23.Homélies sur l'Évangile de saint Jean X, 9, p. 570-571.

24.Homélie II, 1, p. 188.

25.A.G. HOLDER, « New Treasures and Old in Bede's 'De Tabernaculo' and 'De Templo' ", Revue Bénédictine, 99/3-4, 1989, p. 237-249. Le De Tabernaculo et le De Templo ont été édités par D. HURST (CCSL 119A).

26.A. THACKER, « Bede's Ideal of Reform », dans P. WORMALD, D. BULLOUGH, R. COLLINS éd., Ideal and Reality in Frankish and Anglo-Saxon Society. Studies presented to J. M. Wallace-Hadrill, Oxford, 1983, p. 130-153 (p. 133).

27.En particulier : S. DE GREGORIO, « 'Nostrorum Socordiam Temporum': The Reforming Impulse of Bede's later Exegesis », Early Medieval Europe, 11/2, 2002, p. 107-122 ; ID., "Bede's In Ezram et Neemiam and the Reform of the Northumbrian Church », Speculum, 79/1, 2004, p. 1-25. P. MEYVAERT (" The Date of Bede's In Ezram and His Image of Ezra in the Codex Amiatinus », Speculum, 80, 2005, p. 1087-1133) apporte de solides arguments pour situer l'In Ezram parmi les premières œuvres de Bède, ce qui s'oppose à la vision évolutive de S. De Gregorio. Mais il ne conteste pas l'engagement réformateur de cette œuvre.

28.D. IOGNA-PRAT, qui fait commencer le mouvement de sacralisation du lieu de culte à l'époque carolingienne (La Maison-Dieu..., op. cit), a surtout souligné les allégorisations de Bède («Lieu de culte et exégèse liturgique à l'époque carolingienne », dans C. CHAZELLE et B. VAN NAME EDWARDS, The Study of the Bible in the Carolingian Era, Turnhout, 2003,

p. 215-244 [p. 222-223]).

29.PL 100, 773.

30.In Matthaeum, éd. cit., p. 547.

31.PL 106, 1432.

32.0p. cit., p. 1026, 1. 3110 sq.

33.MGH, Leg. I, p. 64.

34.MGH, Cap. episc. 2, p. $111 ; 3$, p. 136.

35.PL 106, 147-149.

36. HINCMAR DE REIMS, De cavendis vitiis et virtutibus exercendis, D. NACHTMANN éd., Munich, 1998, en part. p. 208-209.

37. Walahfrid Strabo's Libellus de exordiis et incrementis quarundam in observationibus ecclesiasticis rerum. A translation and Liturgical Commentary, A.L. HARTING-CORREA éd., LeydeNew York-Cologne, 1996, 10-11, p. 84-88. 
38.MGH, Conc. III, p. 161.

39.. Y. CONGAR, " "L’Église, ce ne sont pas les murs, mais les fidèles" ", dans ID., Sacerdoce et laïcat devant leurs tâches d'évangélisation et de civilisation, Paris, 1965, p. 295-303 (p. 299). 40.De dispensatione ecclesiasticarum rerum, dans Agobardi Lugdunensis opera omnia, L. VAN ACKER éd., Turnhout, 1981 (CCCM 52), p. 130. Sur ces différents textes : M. RUBELLIN, «Biens et revenus ecclésiastiques : la doctrine des évêques carolingiens (milieu VIII ${ }^{\mathrm{e}}$ milieu IX ${ }^{\mathrm{e}}$ siècle) », dans L'Hostie et le denier. Les finances ecclésiastiques du haut Moyen Âge à l'époque moderne, Genève, 1991, p. 25-36 [repris dans M. RUBELLIN, Église et société chrétienne d'Agobard à Valdes, Lyon, 2003, p. 119-130].

41.MGH, Concilia, II, 2, p. 762.

42.PL 142, 1284-1285.

43.J. FEARNS éd., Turnhout, 1968 (CCCM 10), p. 60-61.

44.Opusculum contra hereticos, Madrid, BN 6911, f. 78v. Je remercie Claire Goiran de m'avoir indiqué ce texte qu'elle a édité dans sa thèse : Les Écrits antihérétiques fin XII ${ }^{e}$-début XIII siècle. L'apport de l'édition de trois traités (Madrid, B.N. 6911, Reims, B.M. 495, Troyes, B.M. 1068), Thèse de doctorat, Université Nice-Sophia Antipolis, 2007, II, p. 74.

45.Cités par M. LAUWERS, Naissance du cimetière..., op. cit., p. 340, n. 26.

46. Thomae de Chobham Summa confessorum, F. BROOMFIELD éd., Louvain-Paris, 1968, p. 268 sq . (même si l'auteur concède finalement, contre l'exemple du Christ, et au nom d'une forme de réalisme, la possibilité de pratiquer des activités séculières dans le cimetière et les églises).

47.A. TRIVELLONE, Les Hérétiques imaginés. Iconographie de l'hérésie en Occident avant l'Inquisition (VIII ${ }^{e}$-début XIII ${ }^{e}$ siècle), à paraître, chap. 2.

48.New York, Morgan Pierpont Library M. 492, f. 84r. Sur cette image : R.H. RouGH, The Reformist Illuminations in the Gospels of Matilda, Countess of Tuscany. A Study in the Art of the Age of Gregory VII, La Haye, 1973, ainsi que la notice (n²5) de G.Z. ZANICHELLI, dans A.C. QUINTAVALLE, Wiligelmo e Matilde. L'officina romanica, Parme, 1991, p. 535-544. Je remercie Alessia Trivellone de m'avoir fait connaître l'image et la bibliographie.

49.Adversus simoniacos, MGH, Ldl., I, p. 120, 122, 124, 129, 131, 134, 223.

50.Ibid., p. 196 sq. Cette réflexion a été initiée dans l'Epistula Widonis ad archiepiscopum mediolanensem (ibid., p. 6), et se poursuit à la génération suivante.

51.Adversus simoniacos I, 3, p. 106. Un raisonnement semblable est repris quelques décennies plus tard par Geoffroy de Vendôme, qui l'étend à l'investiture laïque : MGH, $L d l$, II, p. 690. J. LECLERCQ, « Simoniaca heresis », p. 526-528, cite d'autres témoins de cette réflexion. Dans tous les cas, cela reste indépendant de l'utilisation de la péricope biblique. 52.Apologeticus, VIII, MGH, Ldl, II, p. 67.

53.Die Briefe des Petrus Damiani, K. REINDEL éd., MGH, Epistolee IV/1, 1983, p. 143-144, 201, 388.

54.Ibid, 1, p. 472-473; 3, p. 536.

55.J.A. HARRIS (The Place of the Jerusalem Temple in the Reform of the Church in the Eleventh Century, PhD, Toronto, 2002, p. 173, 177-209) a cherché à montrer que le discours réformateur de Pierre s'ancrait dans une réflexion sur le Temple, qui l'aurait conduit à recourir régulièrement à la métaphore des marchands chassés du Temple. Il me semble que cette position, en ce qui concerne la péricope évangélique, doit être nuancée. Je n'ai trouvé que sept occurrences de l'épisode dans quatre lettres seulement (op. cit., 1, p. 143, $201,388,472,501 ; 3$, p. 79, 536), et aucune dans les sermons.

56.J. GILCHRIST, “'Simoniaca Haeresis' and the Problem of Orders... » 
57.CH. DE MIRAMON, « Spiritualia et Temporalia... », p. 267-271.

58. À vrai dire, il existe au moins un cas antérieur à cette lettre dans une charte de 1063 à propos de Saint-Étienne de Nevers, où le passage biblique est appliqué aux prébendes et à l'ensemble des biens temporels (Recueil des chartes de l'abbaye de Cluny, A. BERNARD, A. BRUEL éd., IV, Paris, 1888, p. 489-490). Il est intéressant de noter que cela se situe dans un milieu suburbain de chanoines réguliers, et concerne au premier chef les prébendes, deux aspects sur lesquels insiste $\mathrm{Ch}$. de Miramon.

59.PL 151, 531. Dans les Tractatus in Johanem, X, 6, Augustin dénonce les hérétiques qui, tels des marchands, revendiquent chacun la meilleure marchandise : « O quot proposita fecerunt ", etc. Urbain semble comprendre propositum comme prepositura (intendance). Ainsi, les hérétiques vendeurs de colombes ne vendraient pas leur « projet ", mais leurs produits matériels ; la vente des colombes désignerait donc la vente des produits matériels. Commentaire de cette lettre par Ch. DE MIRAMON, «Spiritualia et Temporalia... ", op. cit., p. 275-277.

60.Ad Gebehardum liber, MGH, Ldl, I, p. 346.

61.R.E. REYNOLDS, The Ordinals of Christ from their Origins to the Twelfth Century, Berlin-New York, 1978, p. 100-102. Ce changement se diffuse ensuite à tous les ordres du Christ. 62.Munich, Bayerische Staatsbibliothek, Clm 14000 (reproduction dans J. HUBERT, J. PORCHER, W.F. VOLBACH, L'Empire carolingien, Paris, 1968, pl. 235).

63.Würzburg, Universitätsbibliothek (reproduction dans G.B. TATUN, « The Paliotto of Sant'Ambrogio at Milan », The Art Bulletin, 26/1, 1944, p. 25-47, fig. 39).

64.Munich, Bayerische Staatbibliothek, Clm. 4453, f. 120v (reproduction dans « The Paliotto... ", fig. 33).

65.PL 161, 714.

66.Le texte d'Augustin est introduit par la formule « Possem dicere... » (je pourrais dire), qui est suivi quelques lignes plus loin par « non ea dico » (mais je ne le dis pas) précision omise par Yves de Chartres! En revanche, les autres textes cités sont conformes aux écrits d'Augustin, Contra Litteras Petiliani Libri Tres, Paris, 1967 (Bibliothèque Augustinienne 30), p. 247 sq.

67.L'affirmation de ce pouvoir de persecutio est un élément essentiel des constructions canoniques : A. STICKLER, « Il potere coattivo materiale della chiesa nella riforma gregoriana secondo Anselmo di Lucca », Studi Gregoriani, 2, 1947, p. 235-285 ; K.G. CUSHING, Papacy and Law in the Gregorian Revolution. The canonistic Work of Anselm of Lucca, Oxford, 1998, p. 122-141, 179-200.

68.GUillaume DE TYR, Chronique, R.B.C. HUYGEns éd., Turnhout, 1986 (CCCM 63), p. 132. 69. C'est l'interprétation nouvelle (l'image elle-même n'étant pas très claire) qu'a proposée N. KENAAN-KEDAR, « The Figurative Western Lintel of the Church of the Holy Sepulchre in Jerusalem ", dans V.P. Goss éd., The Meeting of Two Worlds. Cultural Exchange between East and West during the Period of the Crusades, Kalamazoo, 1986, p. 123-131. 70.BERNARD DE CLAIRVAUX, Éloge de la nouvelle chevalerie, P.-Y. ÉMERY trad., Paris, 1990 (SC 367), 9, p. 75.

71.A. GRABOÏs, « La fondation de l'abbaye du Templum Domini et la légende du Temple de Jérusalem au XII ${ }^{\mathrm{e}}$ siècle ", dans M. BALARD éd., Autour de la première croisade, Paris, 1996, p. 231-237.

72.JEAN DE WÜRZBURG, Descriptio terrae sanctae, texte cité dans M. DE VOGÜÉ, Les Églises de la terre sainte, Paris, 1860, p. 283.

73.CH. DE MIRAMON, « Spiritualia et Temporalia... ». 
74.H. E. J. COWDREY, « Simon Magus in South Italy ».

75.THOMAS DE CHOBAM, op. cit., p. 414, ou JEAN D'ERFURT (N. BRIESKORN, Die Summa Confessorum des Johannes von Erfurt, Francfort-Berne-Cirencester, 1980, II, p. 80).

76.Par exemple, NICOLAS DE GORRAN, In quatuor evangelia commentarius, Anvers, 1618,

p. 186-187.

77.Par exemple, HUGUES DE SAINT-CHER, Postille sur Jean, Venise, 1703, p. 282.

78.Par exemple, HUGUES DE SAINT-CHER, Postille sur Luc, p. 248.

79.Par exemple, THOMAS D'AQUIN, In Mattheum, R. CAI éd., Rome-Turin, 1951, p. 263.

80.Notamment avec l'introduction de la distinction entre le saint des saints et le parvis du Temple ; par exemple, HUGUES DE SAINT-CHER, Postille sur Luc, p. 248.

81.Notamment dans les commentaires de Bonaventure sur Luc (Quaracchi, 1895, p. 498-499), d'Albert le Grand sur Matthieu (Aschendorff, 1987, p. 514-515), et de Thomas d'Aquin sur Jean (R. CAI éd., Rome-Turin, 1952, p. 75-76).

82.Homélie 38, PG 56, 839-843. En fait le texte du Decret est un montage d'un passage de l' Opus imperfectum, composé de citations littérales, d'omissions, de résumés et de paraphrases, mais le sens du texte-source a été respecté.

83.Decretum, d. 88, c. 11, éd. A. FRIEDBERG, p. 308.

84.G. TODESCHINI, «Linguaggi economici ed ecclesiologia fra $\mathrm{XI}^{\mathrm{e}} \mathrm{XII}^{\mathrm{e}}$ secolo : dai Libelli de lite al Decretum Gratiani », dans G. RosSETTI, G. viTolo éd., Medioevo Mezzogiorno Mediterraneo. Studi in onore di Mario Del Treppo, vol. 1, Naples, 2000, p. 59-87 (p. 65-74) ; ID., I mercanti e il tempio. La società cristiana e il circolo virtuoso della ricchezza fra Medioevo ed Età Moderna, Bologne, 2002, p. 176-178.

85.J. VAN BANNIng, Opus imperfectum in Matthaeum. Prefatio, Turnhout, 1988 (CCSL 87B), p. XVI-XXX, qui cite onze manuscrits, principalement de Germanie, entre le VIII ${ }^{\mathrm{e}}$ et le XI siècle; il indique notamment que notre homélie (38) se trouve dans l'homéliaire de Paul Diacre (p. XXIV), mais c'est une faute de frappe : c'est l'homélie 37 qui s'y trouve (conformément d'ailleurs à ce qu'il indique p. XXIII n. 5) ; cf. R. GRÉGOIRE, Les Homéliaires du Moyen Âge. Inventaire et analyse des manuscrits, Rome, 1966, p. 77.

86.Par exemple CASSIODORE, Expositio psalmorum, M. ADRIAEN éd., Turnhout, 1958 (CCSL 97), p. 634-635 (à propos de Ps 70, 15, d'ailleurs cité dans l'Opus imperfectum).

87.G. TODESCHINI, I mercanti e il tempio...

88.BnF lat. 3892, f. $217 \mathrm{v}-218 \mathrm{r}$.

89.Par exemple, f. 96 r sur d. 88 , c. 10.

90.F. 339-340.

91.Éd. cit., p. 301-304. Sur ce texte : O. LANGHOLM, The Merchant in the Confessional. Trade and Price in the Pre-Reformation Penitential Handbooks, Leyde-Boston, 2003, p. 29-30.

92.J.W. BALDWIN, The Medieval Theories of the Just Price. Romanists, canonists and theologians in the 12th and 13th centuries, Philadelphie, 1959, p. 38-42.

93.Op. cit., p. 68, 110, 248, 282.

94.Op. cit., p. 186, 409, 746, 841.

95.BM Reims 162.

96.BnF lat. 625 , f. $151 \mathrm{v}-152$.

97.G. TODESCHINI, I mercanti e il tempio..., p. 174 sq.

98. Nous avons étudié des sermons portant sur le verset Domus mea domus orationis vocabitur.

99.BnF lat. 16473 , f. $138 \mathrm{v}-139 \mathrm{r}$.

100.Rome, Angelica 158, f. 55-56. 
101.10 dimanche après la Trinité, $2^{\mathrm{e}}$ sermon, dans GUILLAUME D'AUVERGNE, Opera omnia, II, Paris, 1674 (reproduction Minerva, Francfort, 1963), p. 320.

102.Sermones dominicales, J.-G. BOUGEROL éd., Grottaferrata, 1977, sermon 36, p. 379-381.

103.Sermons de diversis I, J.-G. BOUGEROL éd., Paris, 1993, sermon 31, p. 391-396.

104.Rome, Casanatense, 4560, f. 395r. sq.

105.Vatican, Ottob. Lat. 557, f. 246v-247r.

106.BnF lat. 15941, f. 19r-21v.

107.Sermon 27, dans S. DELMAS, Eustache d'Arras (o.f.m.) dans les débats universitaires de la seconde moitié du XIII ${ }^{e}$ siècle, Thèse de doctorat, Université Lyon II-Louis Lumière, 2006, p. 347-350 ; je remercie Sophie Delmas de m'avoir communiqué son travail.

108.0p. cit. p. 320.

109.Sermon 29, thèse citée, p. 358.

110.Je ne fais ainsi qu'élargir la belle conclusion de G. LE BRAS, « Les Écritures dans le Décret de Gratien », Zeitschrift der Savigny-Stiftung für Rechtsgeschichte, Kanonistische Abteilung, 27, 1938, p. 47-80 (p. 79-80).

\section{RÉSUMÉS}

Cet article étudie quelques utilisations de la péricope des marchands chassés du Temple entre le $\mathrm{VI}^{\mathrm{e}}$ et le $\mathrm{XIII}^{\mathrm{e}}$ siècle. Il montre d'abord son rôle dans la constitution d'un discours valorisant le lieu de culte. Dans un second temps, il analyse l'évolution des utilisations de ce passage à l'époque de la réforme grégorienne : de la lutte contre la simonie à l'appel à la réforme de l'Église ou aux croisades. Enfin, il analyse l'émergence de la problématique des activités commerciales à partir $\mathrm{du} \mathrm{XII}^{\mathrm{e}}$ siècle. Le but de cet article est de comprendre la logique de la construction de ces différents usages de la Bible. Sa méthode consiste à distinguer commentaires bibliques et usages de la Bible. Il entend montrer la dépendance des usages par rapport aux commentaires.

The Cleansing of the Temple: Commentaries and Social Uses. This article deals with the uses of the pericop of the cleansing of the Temple, between the sixth and the thirteenth centuries. It first shows the part it played in the development of a discourse which enhances the places of worship, then turning on to study how the uses of this episode evolve at the period of the Gregorian reform, from the fight against simony to the call for reform and crusades. What is then analysed is the emergence of the problem of commercial activities from the twelfth century on. The aim of this essay is to understand the logic of the development of these different uses of the Bible. The method I used consists in distinguishing commentaries upon the Bible on the one hand and uses of the Bible on the other, in order to show how uses depend upon commentaries.

\section{INDEX}

Mots-clés : églises, marchands, simonie

Keywords : churches, merchants, simony 


\section{AUTEUR}

\section{EMMANUEL BAIN}

Cepam, UMR 6130, Université de Nice Sophia Antipolis/CNRS, 98, boulevard Édouard Herriot, BP 3209, F-06204 Nice cedex 3 